\title{
Pre-Construction Progress of Giant Steerable Science Mirror for TMT
}

\author{
Yang Fei ${ }^{*}{ }^{1}$, Zhao Hongchao ${ }^{1}$, An Qichang ${ }^{1}$, Guo Peng ${ }^{1}$,Jiang Haibo ${ }^{1}$, Han Linchu ${ }^{1}$, Virginia Ford ${ }^{2}$, \\ Glen Cole ${ }^{2}$, Byron $\mathrm{Smith}^{3}$, Marvin Campbell ${ }^{4}$ \\ 1.Changchun Institute of Optics, Fine Mechanics and Physics, the Chinese Academy of Sciences, \\ Changchun 130033, China \\ 2. Thirty Meter Telescope International Organization, Pasadena, CA, USA \\ 3. XDoubleDout LLC, Flagstaff, AZ, USA \\ 4. MF Campbell Consulting, Garland, TX, USA
}

\begin{abstract}
The Changchun Institute of Optics, Fine Mechanics and Physics (CIOMP) team is developing the Giant Steerable Science Mirror (GSSM) for Thirty Meter Telescope (TMT) which will enter the preliminary design phase in 2016 . The GSSM is the tertiary mirror of TMT and consists of the world's largest flat telescope mirror (approximately $3.4 \mathrm{~m}$ X 2.4 $\mathrm{m}$ X 100mm thick) having an elliptical perimeter positioned with an extremely smooth tracking and pointing mechanism in a gravity-varying environment. In order to prepare for developing this unique mirror system, CIOMP has been developing a 1/4 scale, functionally accurate version of the GSSM prototype during the pre-construction phase of GSSM. The prototype will incorporate the same optomechanical system and servo control system as the GSSM. The size of the prototype mirror is $898.5 \mathrm{~mm} \times 634 \mathrm{~mm} \times 12.5 \mathrm{~mm}$ with an elliptical perimeter. The mirror will be supported axially by an 18 point whiffletree and laterally with a 12 point whiffletree. The main objective of the preconstruction phase includes requirement validation and risk reduction for GSSM and to increase confidence that the challenge of developing the GSSM can be met. The precision mechanism system and the optical mirror polishing and testing have made good progress. CIOMP has completed polishing the mirror, the prototype mechanism is nearly assembled, some testing has been performed, and additional testing is being planned and prepared. A dummy mirror is being integrated into the cell assembly prototype to verify the design, analysis and interface and will be used when testing the prototype positioner tilt and rotation motions. The prototype positioner tilt and rotator structures have been assembled and tested to measure each subsystem's jitter and dynamic motion. The mirror prototype has been polished and tested to verify the polishing specification requirement and the mirror manufacturing process. The complete assembly, integration and verification of the prototype will be soon finished. Final testing will verify the prototype requirements including mounted mirror surface figure accuracy in 5 different orientations; rotation and tilt motion calibration and pointing precision; motion jitter; and internally generated vibrations. CIOMP has scheduled to complete the prototype by the end of July 2016 . CIOMP will get the sufficient test results during the pre-construction phase to prepare to enter the preliminary design for GSSM.
\end{abstract}

Keywords: Giant Steerable Science Mirror, Prototype of GSSM, TMT, Large Aperture Telescope

\section{INTRODUCTION}

TMT (Thirty Meter Telescope) will be one of the largest ground-based telescopes including E-ELT (Europe Extremely Large Telescope) and GMT (Giant Magellan Telescope). All three telescopes will have 30 meter class apertures-larger than any existing optical telescopes in the world so far. Each large telescope has unique features. In the case of TMT, the

*E-mail:yangf@ciomp.ac.cn

Ground-based and Airborne Telescopes VI, edited by Helen J. Hall, Roberto Gilmozzi, Heather K. Marshall, Proc. of SPIE Vol. 9906, 99064M · C 2016 SPIE · CCC code: 0277-786X/16/\$18 · doi: 10.1117/12.2232068 
tertiary mirror has the function of relaying the optical beam from secondary mirror to science instruments that are located on Nasmyth platforms that rotate in azimuth with the telescope, but do not tilt in elevation with it. This architecture places stability and precision requirements on the GSSM that are unprecedented considering the large geometrical size of the nearly 4 meter class optic. The tertiary mirror of TMT is called GSSM (Giant Steerable Science Mirror) because this architecture enables many science advantages such as rapid switching between instruments to allow observing transient phenomenon quickly and by multiple instruments. The GSSM is mounted on a support tower that protrudes through the central hole of primary mirror. Figure 1 shows the GSSM location in the TMT. Figure 2 shows the scheme of GSSM.

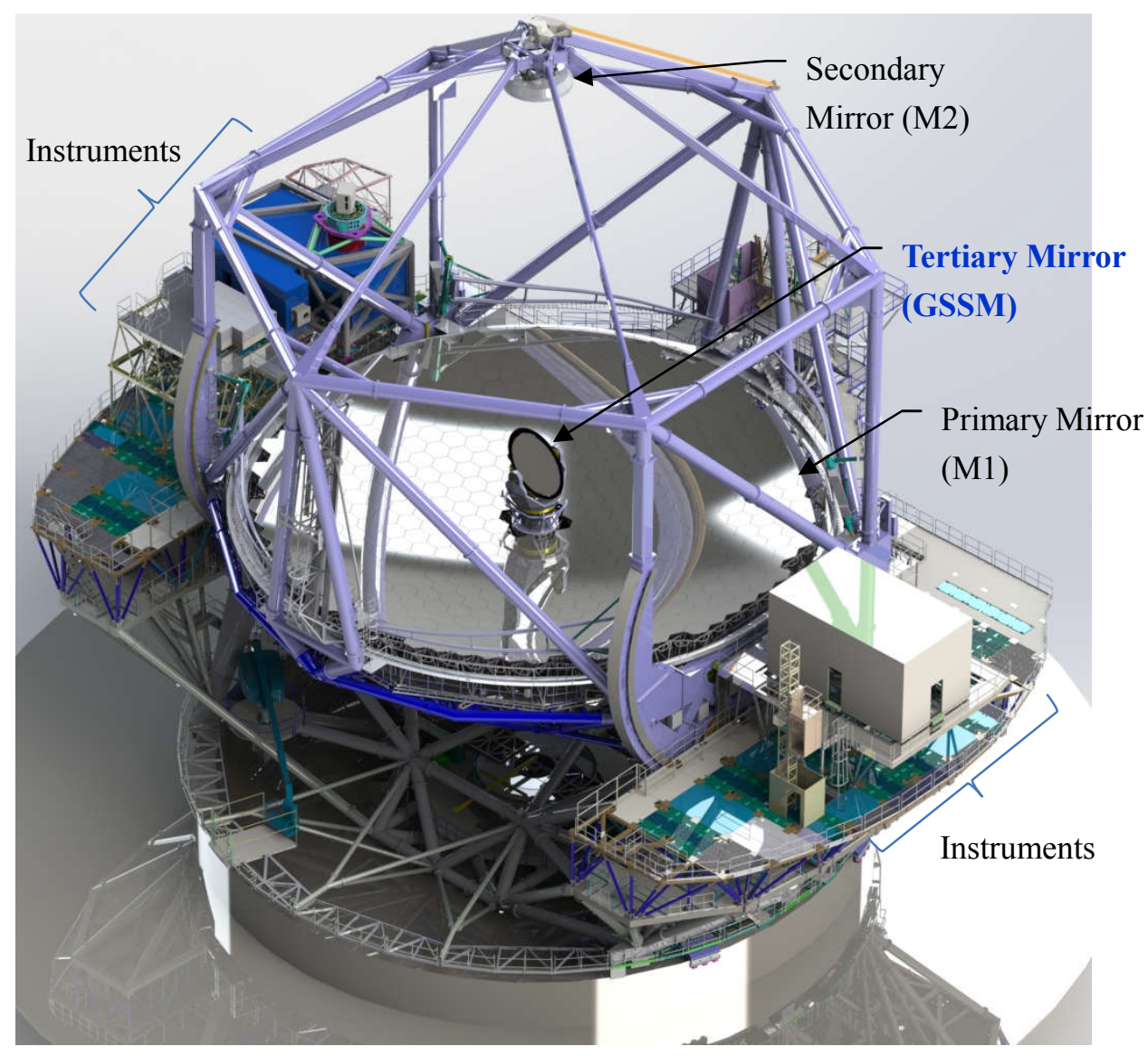

Figure 1. The location of GSSM in TMT 


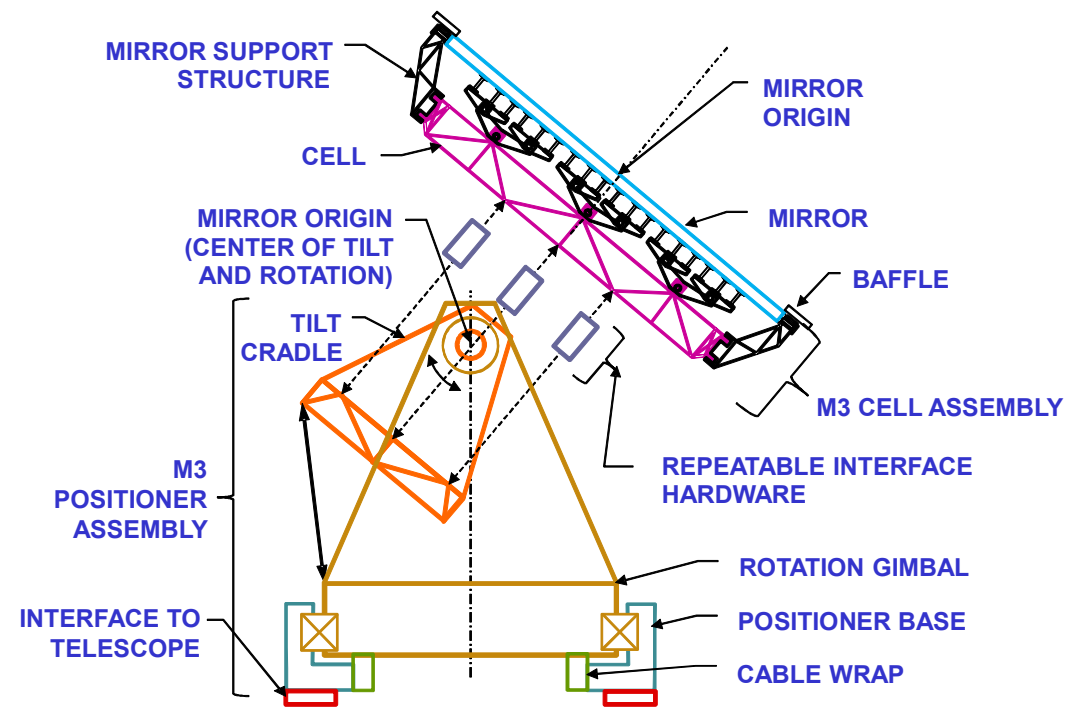

Figure 2. Scheme and configuration of GSSM

\section{DESIGN OF GIANT STEERABLE SCIENCE MIRROR PROTOTYPE}

\subsection{Design of Prototype Cell Assembly (CAP)}

Many support methods were investigated for the Cell Assembly (M3CA) of the GSSM ${ }^{[2]}$. The active support system was employed at first, in which 60 axial actuators supported the tertiary mirror. But it is difficult to decouple GSSM active optics system and M1's. In the end, the active support system was abandoned. Then an 18-point whiffletree system was developed to support the mirror axially, with a 12-point whiffletree system acting as the lateral support. The mirror cell assembly concept design was approved in May 2013, and the concept will be verified in the GSSMP system. Detail design (Figure 3) of the GSSMP CAP was completed in 2015.

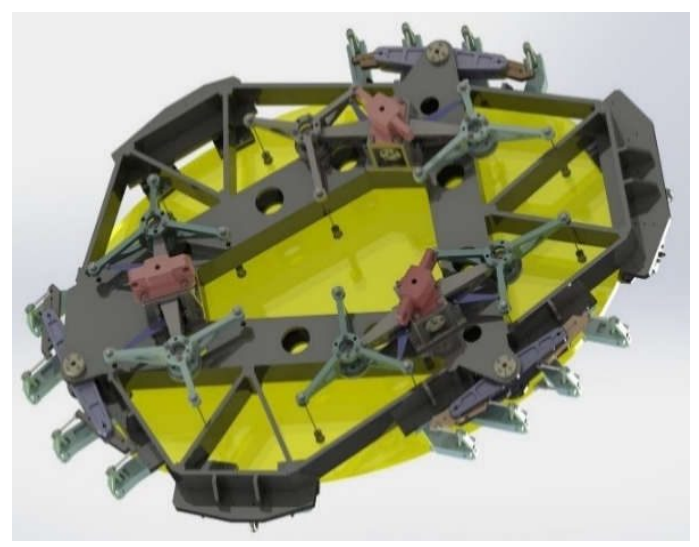

Figure 3.The Detailed Design of M3CAP

\subsection{Design of Prototype Positioner Assembly (PAP)}

The Positioner Assembly (M3PA) is the subsystem of the GSSM which rotates and tilts theM3 Cell Assembly to point the telescope beam towards any selected instrument on either Nasmyth Platform during observing ${ }^{[1][7]}$. This subassembly is responsible for all rigid body motion of the M3 Cell Assembly (M3CA). TheM3PA also rotates and tilts the M3CA to enable servicing and maintenance functions on the Telescope. M3PAP is a prototype model of the M3PA. It has the same 
function as the M3PA. There are two subassemblies in the PAP including of a Rotator and a Tilt Assembly.

The Rotator axis is a challenging subassembly ${ }^{[6]}$. The angle between the Rotator axis and the gravity varies from 0 to $90^{\circ}$. Then the working conditions require that the Rotator axis supports the axial load, lateral load and the tilting moment. This is a challenge to the designer. Three drive solutions were investigated for Rotator drive system. They were direct drive, gear transmission and frictional roller transmission. We chose the direct drive for the M3PAP. The final design of the Rotator structure is illustrated in Figure 4.

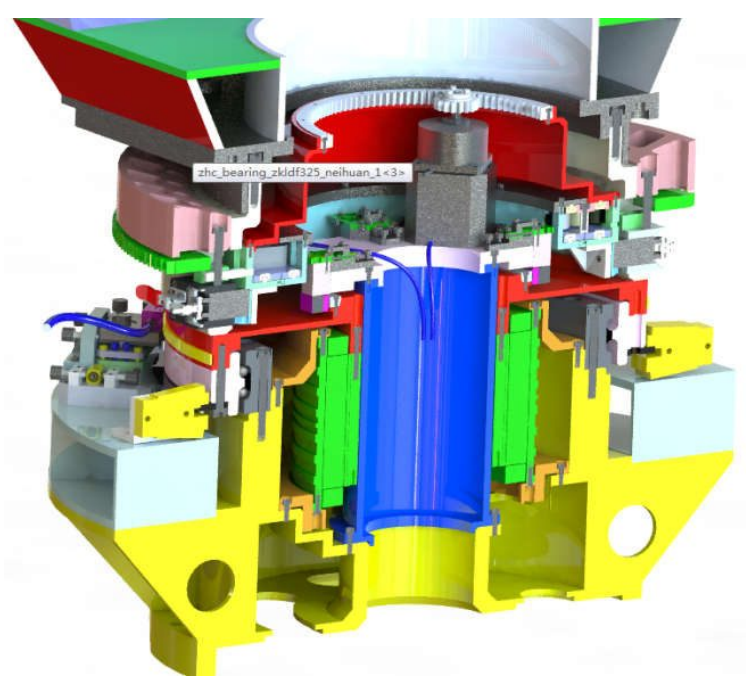

Figure 4.Cross section view of M3PAP Rotator

Due to the volume requirement of GSSM to stay within the obscuration shadow of the M2, the Tilt Assembly needs a compact design ${ }^{[3]}$. In addition, the angle between the Tilt axis and the gravity varies from 0 to $90^{\circ}$. Thus the Tilt axis must withstand the combination load. There are four key components in Tilt Assembly. They are a turntable, a set of axes, a cradle, and the interface with the M3CAP. The turntable is a plate welded structure, while the cradle is a welded truss. The tilt axes are hollow forgings with inner diameters large enough for to contain direct drive motor systems. The Tilt Assembly design details are illustrated in Figure 5.

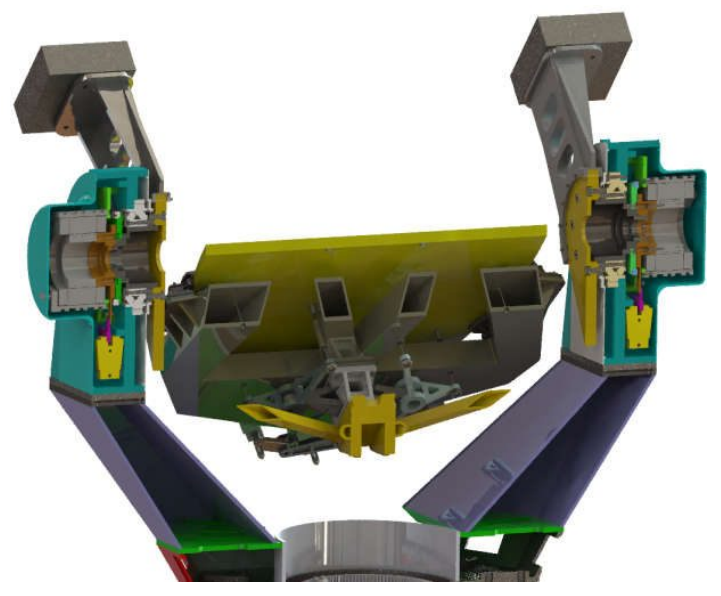

Figure 5. Cross section of the tilt structure

Static analysis and modal analysis were carried out to evaluate M3PAP performance. The structure has been constructed in ANSYS (figure 6). And the other non-structure components are represented by mass-points. 

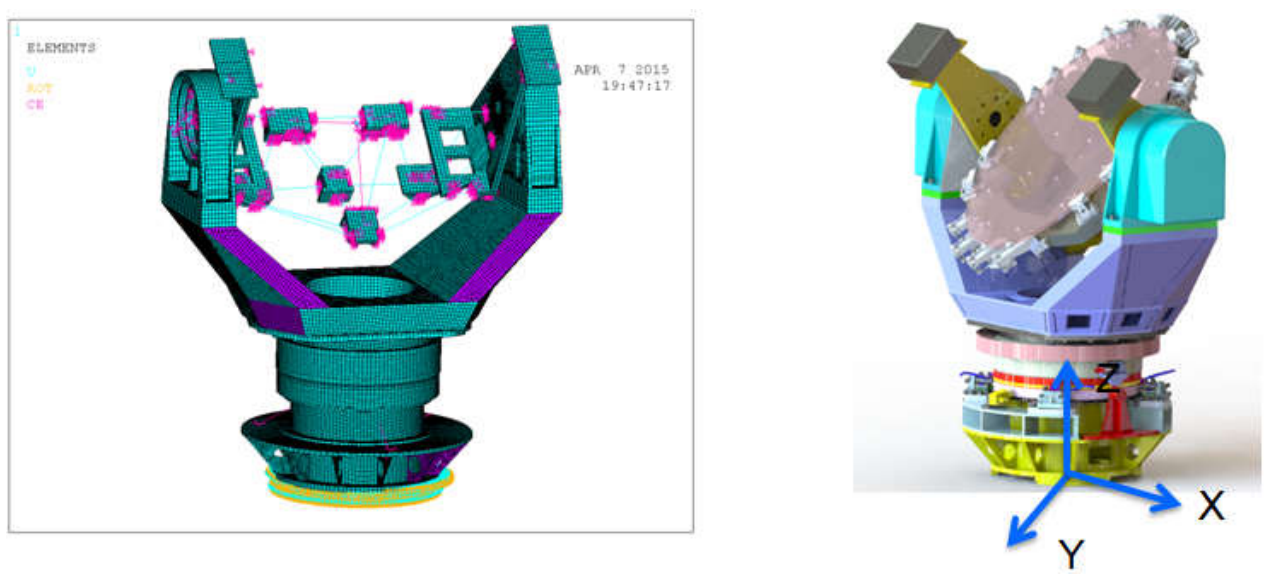

Figure 6. FE Model and 3-D model

Table.1 Modal analysis results

\begin{tabular}{|c|c|c|c|c|c|}
\hline & $\begin{array}{l}\text { Modal } \\
\text { frequency } \mathrm{Hz}\end{array}$ & Modal shape & & $\begin{array}{l}\text { Modal } \\
\text { frequency } \mathrm{Hz}\end{array}$ & Modal shape \\
\hline 1 & 38.53 & Sway in $\mathrm{x}$ direction & 6 & 68.60 & $\begin{array}{l}\text { Cradle bending and yoke } \\
\text { bending }\end{array}$ \\
\hline 2 & 45.27 & Rotate about $\mathrm{z}$ direction & 7 & 109.65 & Too complex \\
\hline 3 & 48.34 & Sway in y direction & 8 & 111.13 & Cradle rotates about $\mathrm{x}$ axis \\
\hline 4 & 56.06 & $\begin{array}{l}\text { Two arms bend in opposite } \\
\text { direction along } \mathrm{x} \text { axis }\end{array}$ & 9 & 123.61 & Too complex \\
\hline 5 & 62.46 & $\begin{array}{l}\text { Complex modal shape combined } \\
\text { by tilt and sway in y direction }\end{array}$ & 10 & 137.73 & Cradle deformation \\
\hline
\end{tabular}

After 4 months fabrication, all the parts were received. Assembly phase started at the beginning of 2016 .

\section{PROGRESS OF GSSMP}

\subsection{CAP progress}

As shown in figure 7, axial pads were glued onto the dummy-mirror in April 2016. A laser tracker was used to assist the process. The location error was controlled to less than $0.1 \mathrm{~mm}$.

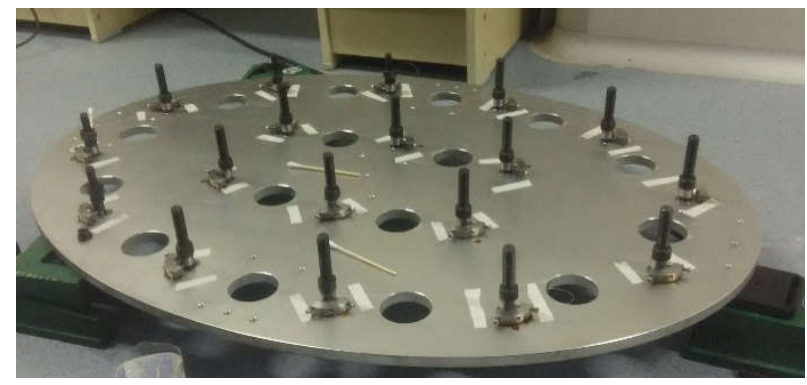

Figure 7. Axial pads in place 


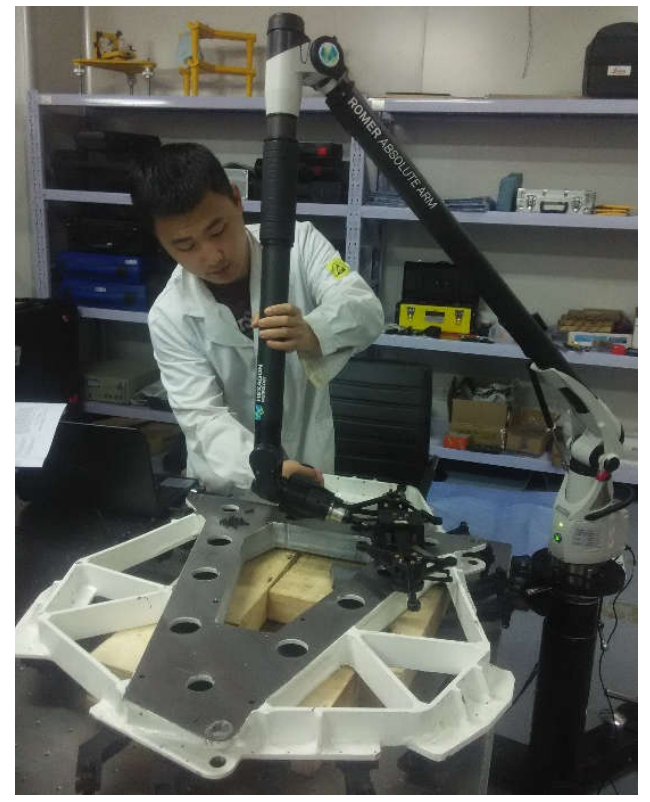

Figure 8. Axial whiffletree installed

Then the designers installed the axial whiffletree structure onto the mirror cell. Using the advantage of the mobile coordinate measuring machine, the accuracy of the 18 axial support points was within $1 \mathrm{~mm}$ as shown in figure 8 . The final assembly of the axial whiffletree mirror support on the Cell structure is shown in Figure 9.

\subsection{Mirror Prototype progress}

The mirror prototype was polished to meet a of SlopeRMS less than $1 \mu$ radian on the polishing support structure. The result of the mirror surface accuracy measured with an on-site subaperture Fizeau interferometer and using sub-aperture stitching technology is shown in figure 9.

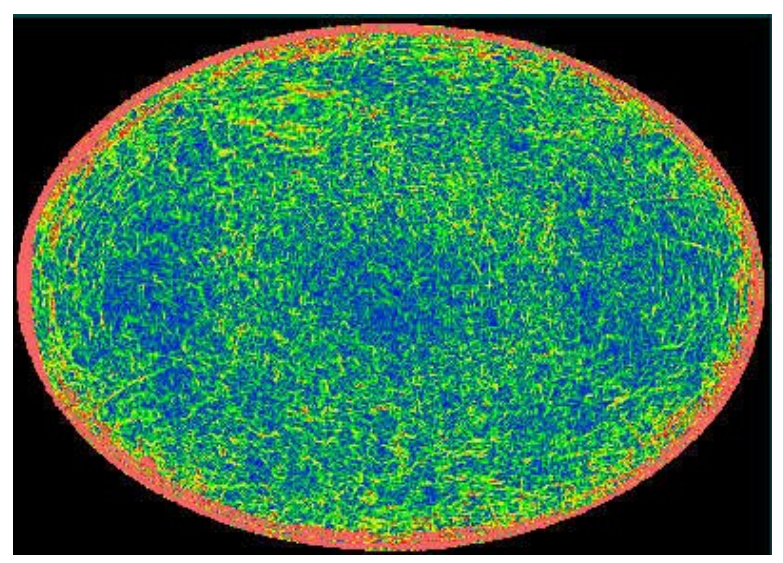

Figure 9. Phasemap of M3MP after polish (low order error removed)

In addition, to measure low order polished mirror error, a scanning pentaprism assembly was developed and used to measure focus and astigmatism of the resulting mirror surface. A photograph of the scanning pentaprism equipment is shown in Figure 10. The mirror easily met its low order requirement. 


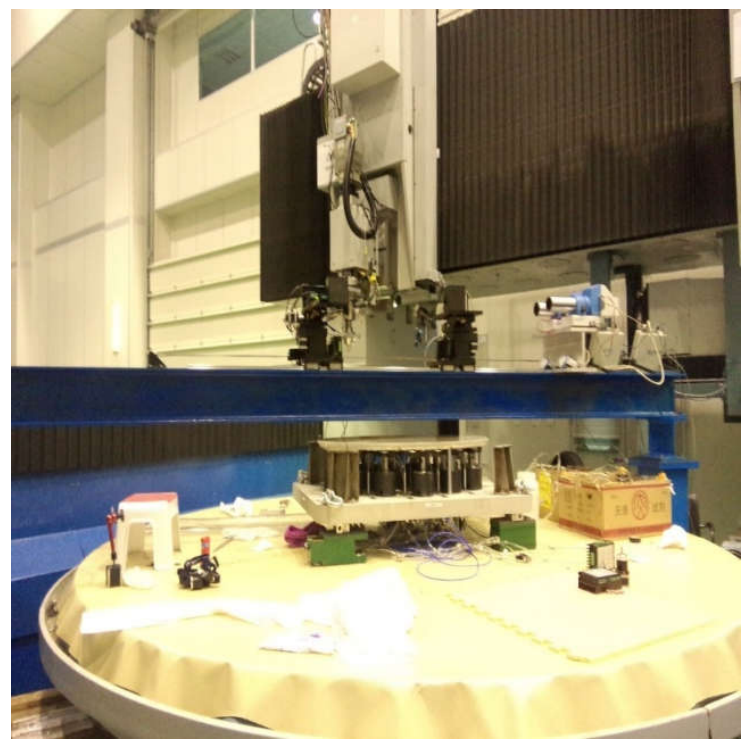

Figure 10. Scanning pentaprism equipment for M3MP

\subsection{PAP progress}

The wobble of the Rotator has been measured to be 1.6 arcseconds after removing the first order component that the TMT telescope is able to filter out. The measure curves can be found in figure 11 .

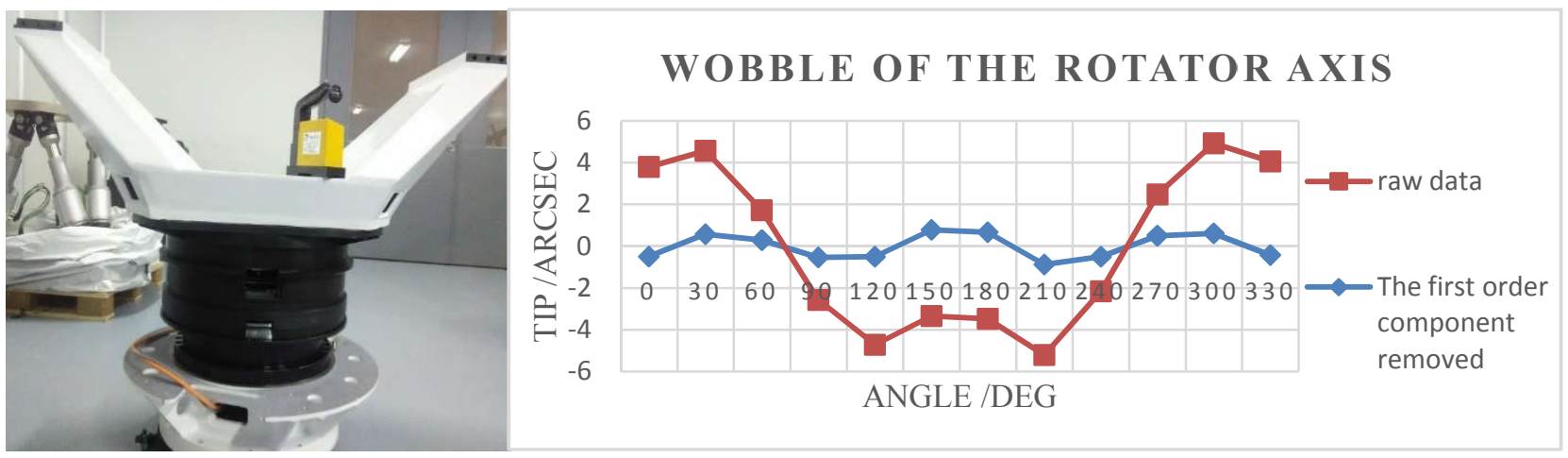

Figure 11. Rotator axis wobble measurement and result 

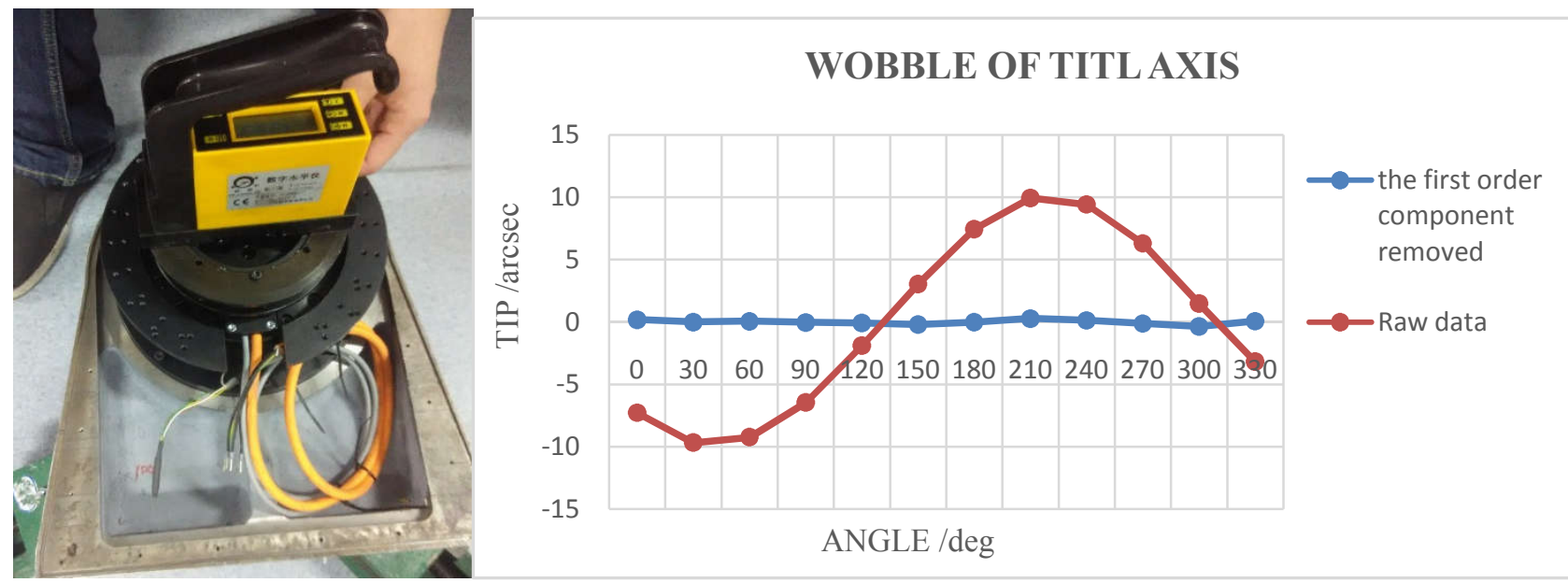

Figure 12. Wobble measurement of Tilt axis and results

A same process was used on the tilt axis. Fortunately, both of the two axes performances meet requirements. The testing data can be found in figure 12 .

In order to ensure the performance of GSSMP, some dynamic tests about the key components have been done. Take the cradle as an example. An impact test was carried out with the cradle suspended. The response can be found in figure 13. The test result is consistent with the FEA. And the first modal frequency is about $78 \mathrm{~Hz}$.

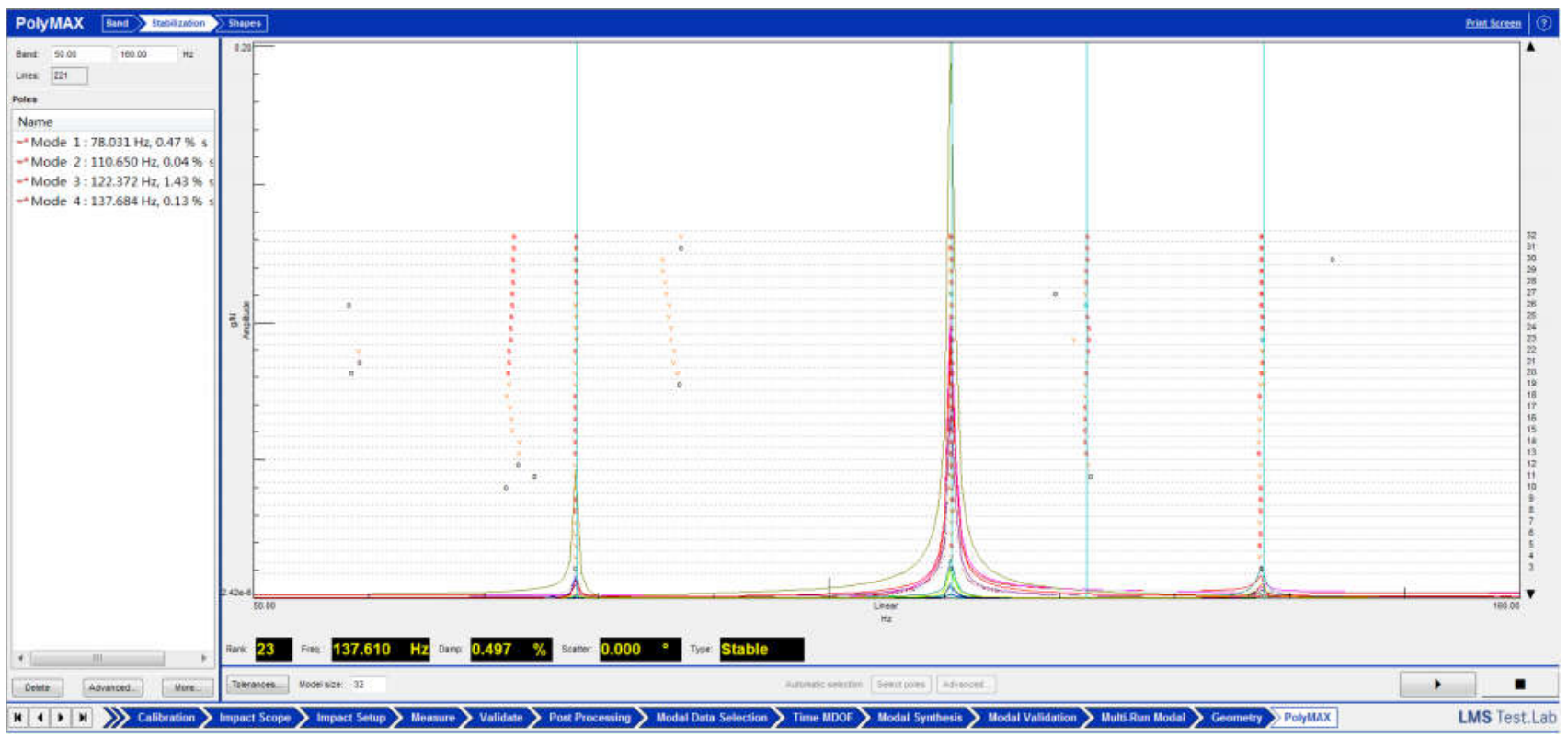

Figure 13. Impact test of the Cradle

The yoke was supported on an isolating foam pad and measured. An accelerometer was bonded tothe yoke to show the characteristics motion of the yoke. The test result is consistent with the FEA. And the first modal frequency is about 241 $\mathrm{Hz}$. 


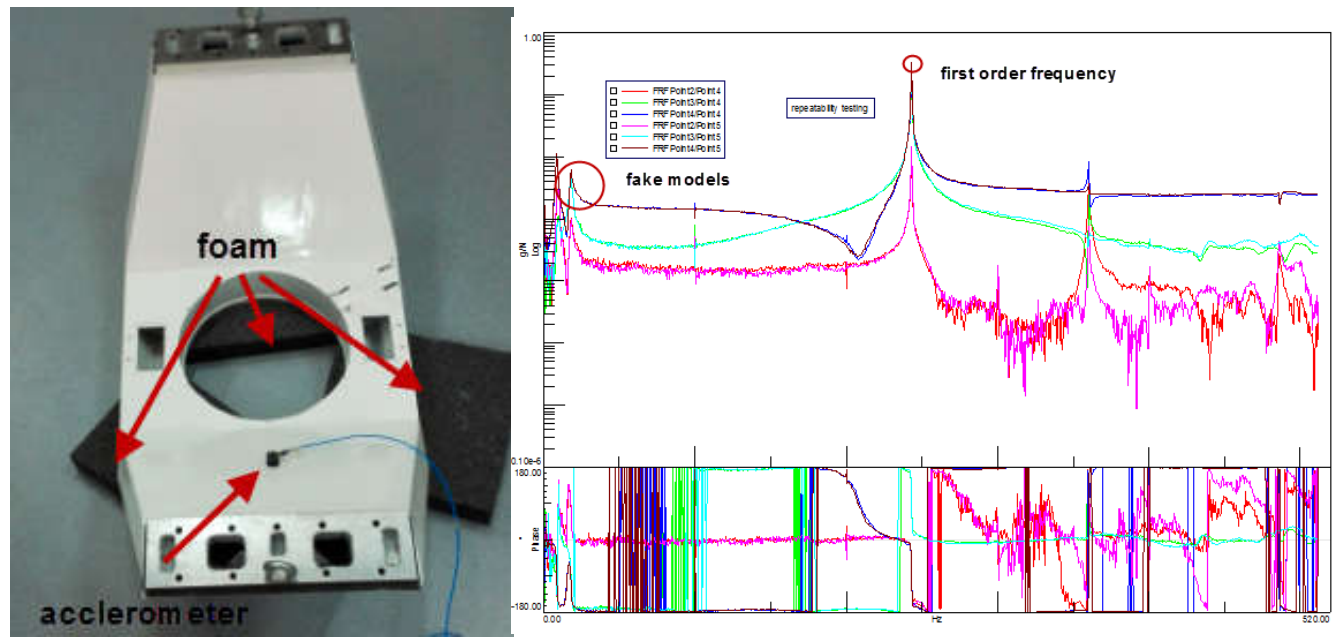

Figure 14. Impact test of the Yoke

The assembled Tilt Assembly is shown in figure 15. There are two tilt axis spindles, a cradle which contains the two spindles that integrate the whole assembly. Due to the mass imbalance of the mirror cell assembly, lead counterweights are installed on the two cradle arms to balance the tilt axis.

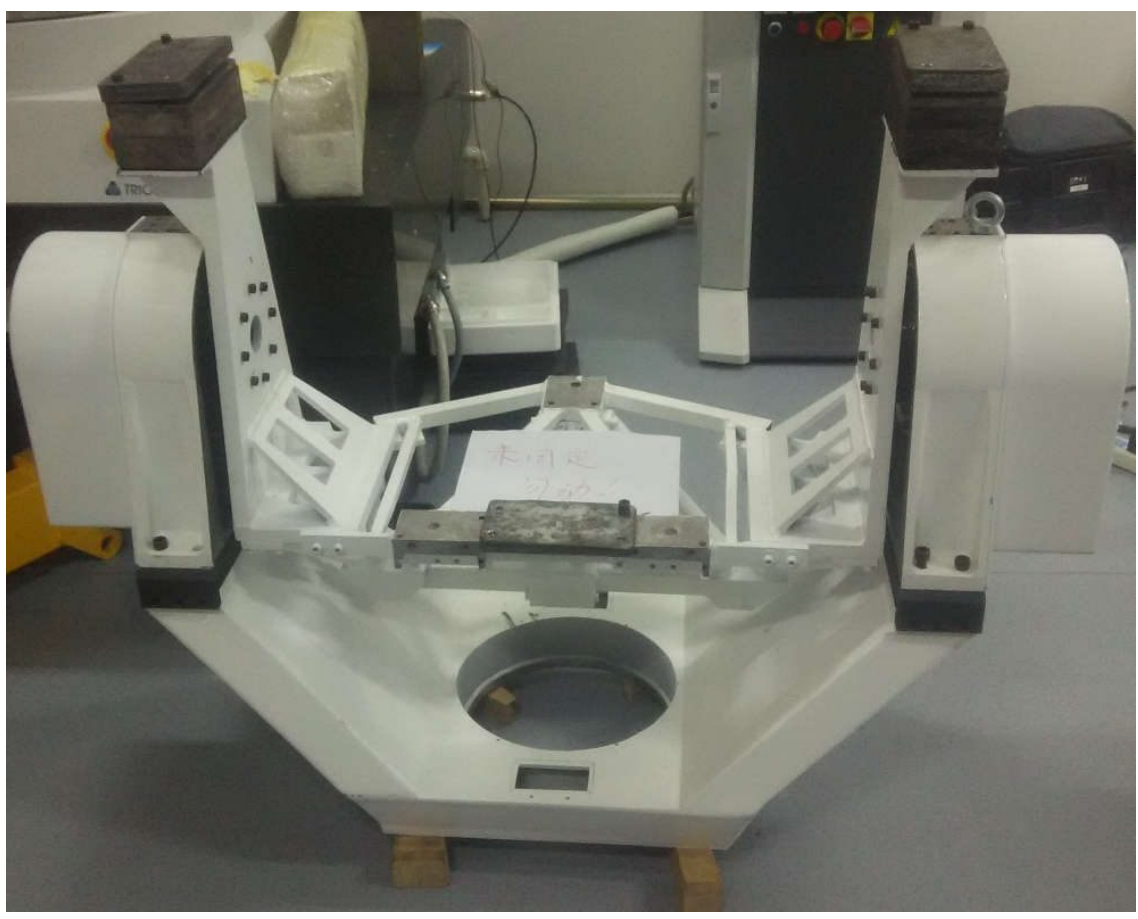

Figure 15.Tilt axis has been completed

\section{TESTING PLANNING}

4.1 Mirror surface figure test

The polished mirror surface figure has been measured in the face up configuration resting on a specially designed test support that duplicates the configuration of the Cell Assembly axial support. In order to determine the influence of the Cell Assembly support on the mirror surface, testing will be performed with the mirror mounted to the Cell Assembly 
in 5 gravity orientations. The same interferometer set-up that was used to measure the face up configuration will be used to measure the mounted mirror surface. A horizontally oriented 24" ZYGO interferometer will be used for the other four orientations which are horizontal. A test fixture to hold and align the mounted mirror has been designed. This fixture is still being fabricated; but a CAD model is shown in Figure 16.

It can translate the CAP in two directions and can tip/tilt the CAP to collimate with the 24" ZYGO interferometer. The weight of the CAP is counterbalanced, so there equipment has locks for safety while the CAP is installed or removed. Also, there will be mark along the guide to monitor the location of the CAP.

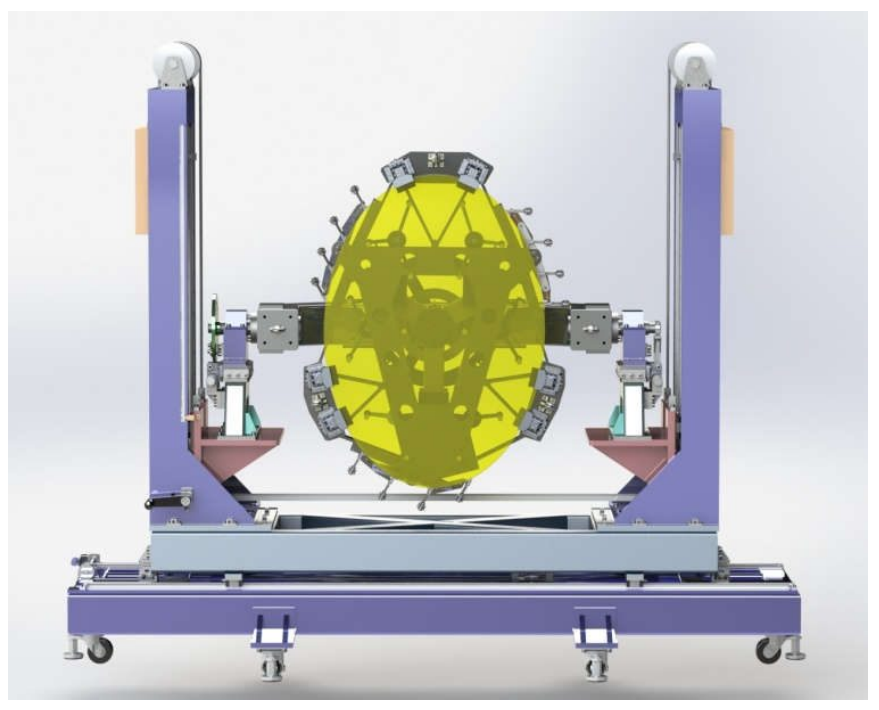

Figure 16. The sketch of the testing fixture
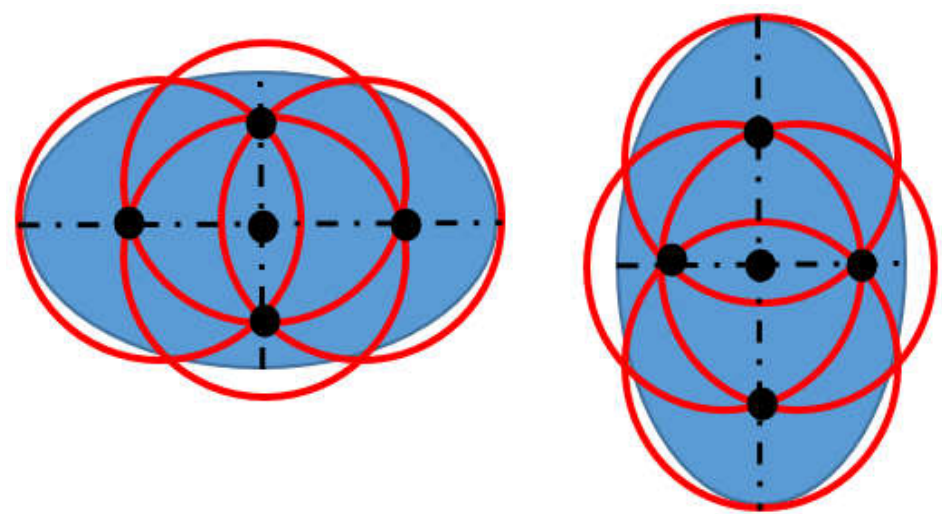

Figure 17.Arrangement of sub-aperture for optical testing and the location of the mark (dark spot)

\subsection{Calibration and motion test}

Pointing precision of the CAP will be measured using a Laser Tracker ${ }^{[4][5]}$. Spherically Mounted Retroreflectors (SMRs) will be mounted to a dummy mirror mounted on the Cell Assembly which reflect the laser beam back to its source from any direction. Mounting hardware for the SMRs are shown in figure 18. The repeatability accuracy of this facility has been measured to be is less than $10 \mu \mathrm{m}$ in the lab with controlled temperature of $22.3^{\circ} \mathrm{C}$. 


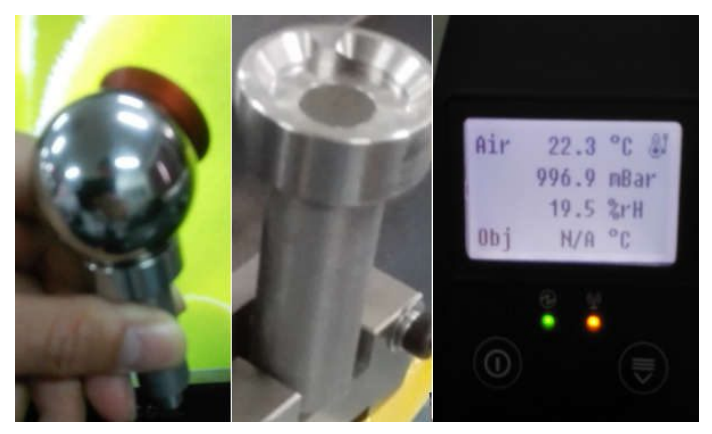

Figure 18. The interface of the laser tracker

During the calibration testing, the GSSMP will be located on a flat testing base, then raised and located on a tilted testing base to understand the influence of gravity. The interface between the GSSMP and the testing base consists of a kinematic arrangement of ball-cone, ball-groove and ball-plane, as shown in Figure 19. As the GSSMP is lowered by crane onto the interface, two guide rods are used to help turn around about the interface 1,the interface 2 will be firmly attached then, as shown on the right side. The last interface to be fastened will be interface 3 .
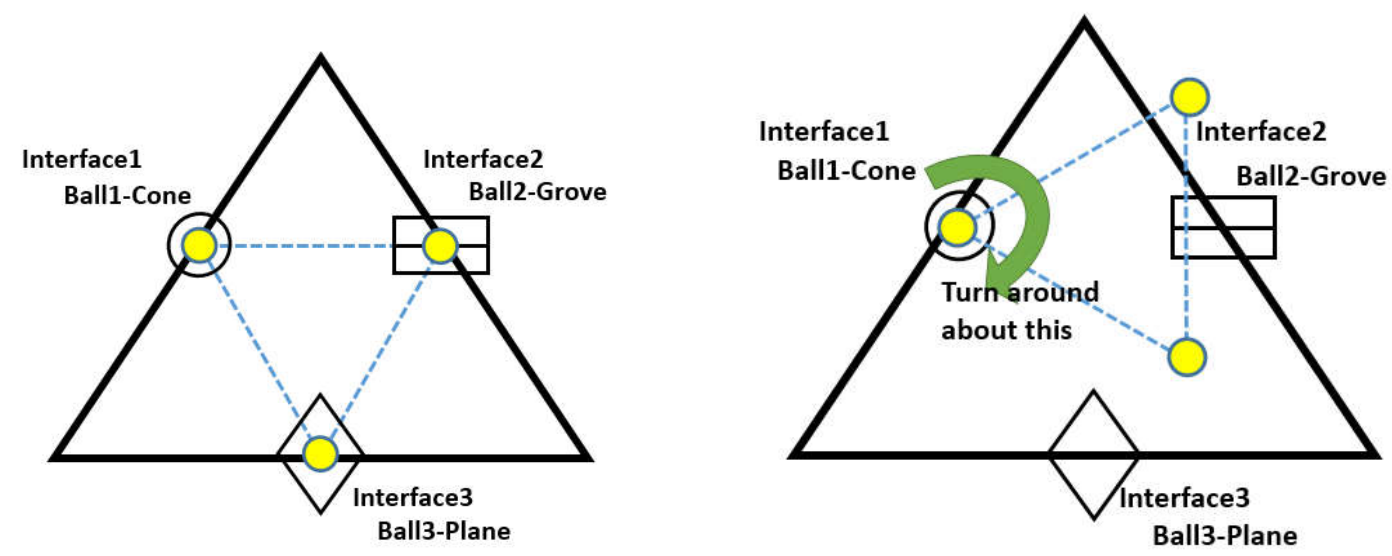

Figure 19. The Kinemics Interface (left) and the Assemble of the Interface(right)

In order to calibrate the motion, laser tracker measurements of the actual mirror position will be made at 1 degree increments that step through the rotation and tilt ranges of motion. The resulting data will be used to create look-up tables that calibrate actual position to encoder readings.

After calibration is performed, then the required motions will be measured using calibrated encoder readings to measure velocity, step size and settling time, and jitter.

\subsection{Vibration testing}

\subsubsection{Modal testing}

The test objective is to validate the FEA results and revise the CAD model according to the following procedure,

1. The dummy mirror will be used in this test

2. The accelerometers will be located as follows: 
- $\quad 4$ on the dummy mirror

- $\quad 3$ on the CAP

- 2 on the Yoke

- 1 on the rotation table

- $\quad 3$ at the interface to the mount fixture

There are two kinds of accelerometers. One has only one axis, and the other can measure acceleration in three directions. There are screw holes in the dummy mirror for the location of the accelerometers.
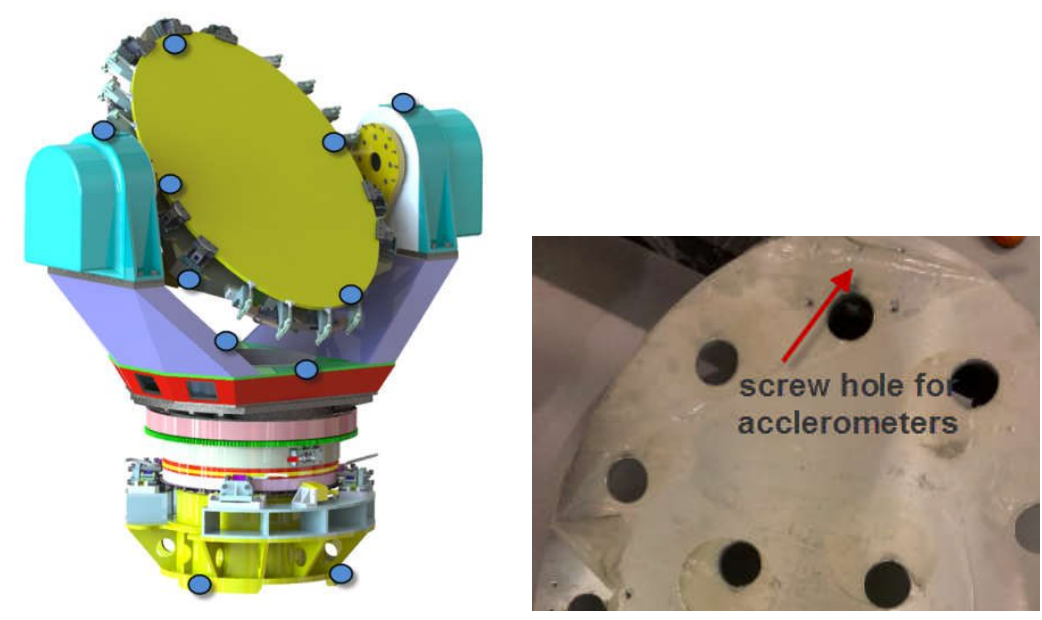

Figure 20. The allocation of the accelerometer for the GSSMP

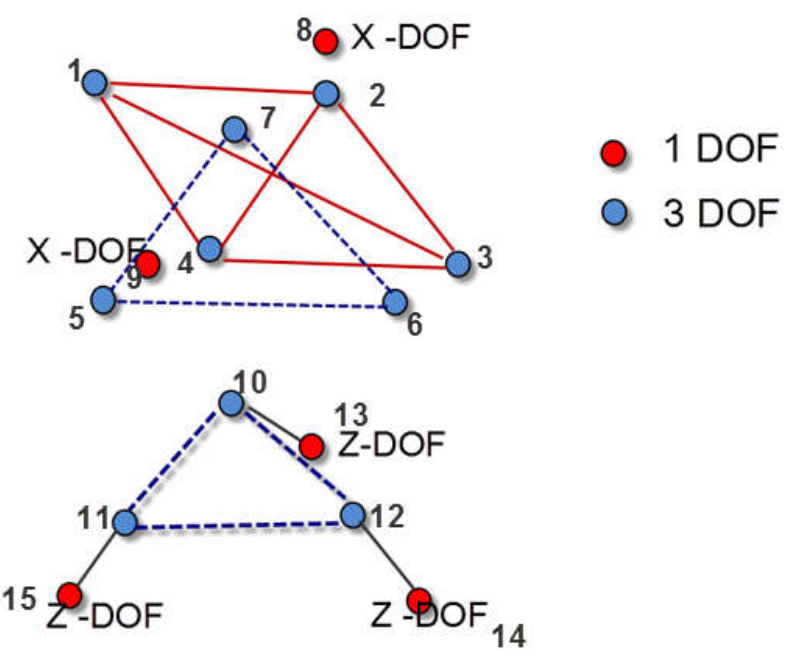

Figure 21. The types and orders of accelerometers on the GSSMP

3. The set-up for the hammer test is the GSSMP mounted to an isolation stand. The sensor cables are attached to the GSSMP with soft plastic foam to prevent parasitic vibrations.

4. The next step is to impact the GSSMP with hammer using the following sequence:

a) Impact close to the accelerometer at point 1 .

b) Remove the accelerometer.

c) Impact at point 1. 
d) Every impact will be repeated for five times to constrain the noise.

e) Attach the accelerometer at point 1 .

5. For points $2 \sim 14$, repeat the steps described in 4 .

6. After the hammer testing, the excitation will be applied to the GSSMP mounted on the isolation stand using an exciter. The excitation points are chosen as 13,14 and 15.

7. For the excitation testing, the wave plot chosen is the fast sin sweep.

4.3.2 Internal vibration testing

The internal vibration requirement is that the force transmitted from the drive systems of the GSSMP to the tower shall be less than $\frac{5}{16} N=0.31 N$. The GSSMP will be mounted to an isolation base for the test.

The following equations calculate the accuracy required for the measurement and the parameters of the test set-up. In order to make the measurement, measurements must have accuracy that is less than $\delta F=0.1 \mathrm{~N}$

Converting,

$F=a \mathrm{~m}$ where theGSSMP is 0.7 tons.The accuracy of the accelerometers must beless than the following:

$$
\frac{\delta F}{m}=\frac{0.1 \mathrm{~N}}{700 \mathrm{~kg}}=0.014 \mathrm{mG}
$$

The system stiffness is then used to estimate the displacement amount.

$F=k x$ the system is 0.7 ton, the frequency is $30 / 2=15 H z$, the stiffness $k=\frac{6 N}{\mu m}$.the accuracy of the displacement metrology facility is $\frac{\delta F}{k}=\frac{0.1 \mathrm{~N}}{6 N / \mathrm{um}}=16 \mathrm{~nm}$

Figure 22 shows a schematic of the test set-up. The mass, stiffness and damping parameters for subsystems 1 and 2 are $m_{1}, k_{1}, c_{1}$ and $m_{2}, k_{2}, c_{2}$

The internal force between 1 and 2 is $f$. With Laplace transform $\mathrm{F}(\mathrm{s})$,

$$
\left\{\begin{array}{c}
m_{1} \ddot{x_{1}}=-\left(x_{1}-x_{2}\right) k_{1}+\left(\dot{x_{1}}-\dot{x_{2}}\right) c_{1} \\
m_{2} \ddot{x}_{2}=-x_{2} k_{2}+\dot{x_{2}} c_{2}+\left(x_{1}-x_{2}\right) k_{1}-\left(\dot{x_{1}}-\dot{x_{2}}\right) c_{1}+f
\end{array}\right.
$$

$x_{1}$ and $x_{2}$ are the displacements of the mass 1 and mass 2, with Laplace transforms $X_{1}(s) 、 X_{2}(s)$

The transfer function of the two subsystems are $T_{1}(s) 、 T_{2}(s)$ 


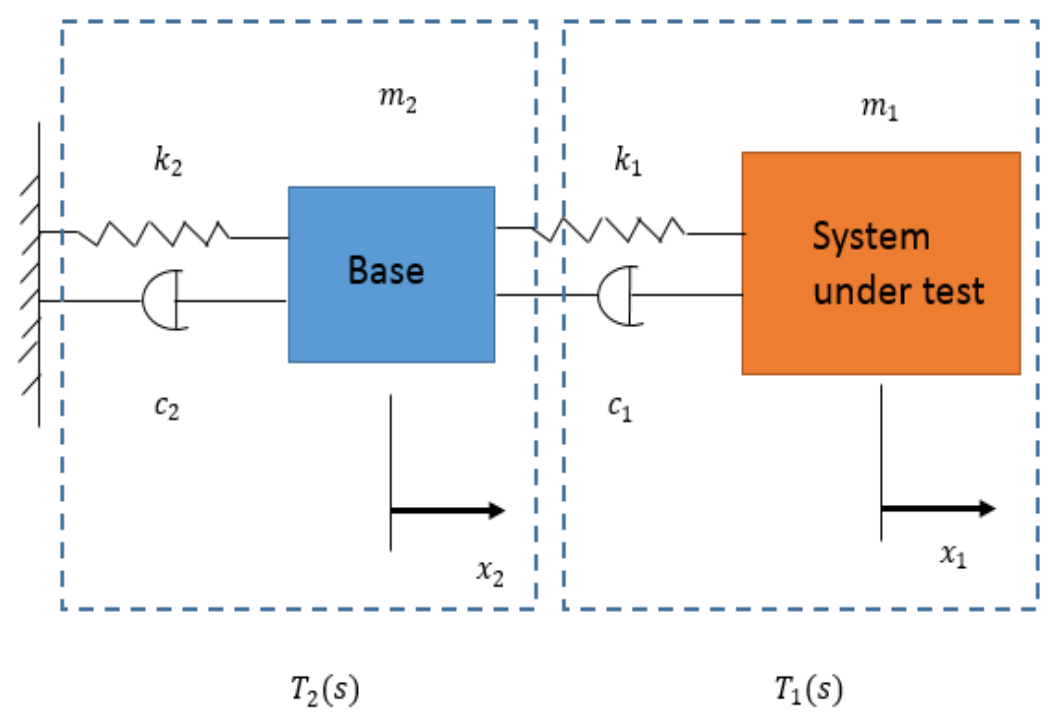

Figure 22. Schematic of the internal vibration test

Laplace transform of the force can be expressed as

$$
\mathrm{F}(\mathrm{s})=-\frac{X_{1}(s)}{T_{1}(s)}-\frac{X_{2}(s)}{T_{2}(s)}
$$

The relationship of $\mathrm{F}(\mathrm{s})$ and Laplace transform of the accelerometer $s^{2} X_{2}(s)$ can be expressed as

$$
\frac{\mathrm{F}(\mathrm{s})}{s^{2} X_{2}(s)}=-\frac{1}{s^{2}}\left(\frac{X_{1}(s)}{T_{1}(s) X_{2}(s)}+\frac{1}{T_{2}(s)}\right)
$$

if $m_{2} \ll m_{1}, k_{1} \gg k_{2}$ then :

$$
\frac{\mathrm{F}(\mathrm{s})}{s^{2} X_{2}(s)}=-\frac{1}{s^{2}}\left(\frac{1}{T_{1}(s)}+\frac{1}{T_{2}(s)}\right)
$$

The transfer function can be determined by the frequency response function and the frequency response function can be reached by the modal testing or FEA model.

\section{CONCLUSION}

Pre-construction of Giant Steerable Science Mirror for TMT is currently under the way to complete final testing by the end of July 2016. To date, the design goals of the prototype and gains from optical manufacturing and mechanical structure assembly are being met.

\section{REFERENCES}

1. DENG Yong-ting, LI Hong-wen, WANG Jian-li. Overview of AC servo control system for the large telescope[J]. Chinese Optics, 2015,8(6): 895-908.

2. Yang F, Zhao H, Guo P, et al. Pre-construction of giant steerable science mirror for TMT[C]/SPIE Optical Engineering+ Applications. International Society for Optics and Photonics, 2015: 95730T-95730T-12.

3. Zhao H, Zhang J, Yang F, et al. Study of tilt axis bearing arrangement for M3S of TMT project[C]//7th International 
Symposium on Advanced Optical Manufacturing and Testing Technologies (AOMATT 2014). International Society for Optics and Photonics, 2014: 92800M-92800M-6.

4. An Q C, Yang F, Guo P, et al. Power spectral density specification and analysis of system optical jitter[C]//International Symposium on Optoelectronic Technology and Application 2014. International Society for Optics and Photonics, 2014: 929814-929814-5.

5. Yang F, An Q C, Wang F G, et al. Analysis of power spectral density as a performance metric for TMT M3[C]//7th International Symposium on Advanced Optical Manufacturing and Testing Technologies (AOMATT 2014). International Society for Optics and Photonics, 2014: 92820C-92820C-6.

6. Su Y, Zhang J, Yang F, et al. Structure performance analysis for TMT tertiary mirror system[C]//7th International Symposium on Advanced Optical Manufacturing and Testing Technologies (AOMATT 2014). International Society for Optics and Photonics, 2014: 92800L-92800L-7.

7. Deng Y, Li H, Yang F, et al. Integrated modeling and dynamics simulation for the TMT-M 3 control system[C]//7th International Symposium on Advanced Optical Manufacturing and Testing Technologies (AOMATT 2014). International Society for Optics and Photonics, 2014: 92800J-92800J-7. 